University of Nebraska - Lincoln DigitalCommons@University of Nebraska - Lincoln

2011

\title{
Colossal negative magnetoresistance in dilute fluorinated graphene
}

X. Hong

S. -H. Cheng

C. Herding

J.Zhu

Follow this and additional works at: https://digitalcommons.unl.edu/physicshong

Part of the Atomic, Molecular and Optical Physics Commons, and the Engineering Physics Commons

This Article is brought to you for free and open access by the Research Papers in Physics and Astronomy at DigitalCommons@University of Nebraska Lincoln. It has been accepted for inclusion in Xia Hong Publications by an authorized administrator of DigitalCommons@University of Nebraska Lincoln. 


\title{
Colossal negative magnetoresistance in dilute fluorinated graphene
}

\author{
X. Hong, ${ }^{1,4}$ S.-H. Cheng, ${ }^{1}$ C. Herding, ${ }^{1,3}$ and J. Zhu ${ }^{1,2}$ \\ ${ }^{1}$ Department of Physics, The Pennsylvania State University, University Park, Pennsylvania, 16802, USA \\ ${ }^{2}$ The Materials Research Institute, The Pennsylvania State University, University Park, Pennsylvania, 16802, USA \\ ${ }^{3}$ Physikalisches Institut, Universität Münster, Wilhelm-Klemm-Strasse 10, D-48149 Münster, Germany \\ ${ }^{4}$ Department of Physics and Astronomy, University of Nebraska-Lincoln, Lincoln, NE 68588, USA \\ (Received 8 October 2010; revised manuscript received 17 December 2010; published 14 February 2011)
}

\begin{abstract}
Adatoms offer an effective route to modify and engineer the properties of graphene. In this work, we create dilute fluorinated graphene using a clean, controlled, and reversible approach. At low carrier densities, the system is strongly localized and exhibits an unexpected, colossal negative magnetoresistance. The zero-field resistance is reduced by a factor of 40 at the highest field of $9 \mathrm{~T}$ and shows no sign of saturation. Unusual staircaselike field dependence is observed below $5 \mathrm{~K}$. The magnetoresistance is highly anisotropic. These observations cannot be explained by existing theories, but likely require adatom-induced magnetism and/or a metal-insulator transition driven by quantum interference.
\end{abstract}

DOI: 10.1103/PhysRevB.83.085410

PACS number(s): 72.80.Vp, 73.20.Fz, 75.47.-m, 73.20.Jc

\section{INTRODUCTION}

Defects are powerful tools to control the electronic properties of graphene. ${ }^{1-7}$ In particular, point defects such as vacancies and adatoms can perturb the electronic states of graphene strongly, leading to midgap states ${ }^{1}$ and a drastic change of transport properties. ${ }^{3-7}$ Theory predicts that point defects could introduce magnetic moments into graphene, ${ }^{8-10}$ and moments occupying the same (opposite) graphene sublattice may interact ferromagnetically (antiferromagnetically) to produce competing magnetic orders. ${ }^{9,10}$ Although previous studies have hinted at weak magnetism in certain disordered $s p^{2}$ carbon materials, ${ }^{11}$ clear evidence of magnetism in singlelayer graphene remains elusive. Magnetic graphene may carry important technological implications. A controllable defect coverage, together with in situ wide tunability of the electronic states, makes defective graphene an ideal venue to explore the above opportunities.

In this work, we report that dilute fluorinated graphene (DFG) shows an unexpected colossal negative magnetoresistance. A perpendicular magnetic field reduces the zerofield resistance of DFG by 40 -fold at $9 \mathrm{~T}$ with no sign of saturation, an effect comparable to those observed in ferromagnetic semiconductors ${ }^{12}$ and manganites. ${ }^{13}$ Furthermore, an unprecedented staircaselike field dependence develops at the lowest temperatures. These striking observations cannot be accounted for by existing theories, but likely require adatom-induced magnetism and/or a metal-insulator transition driven by quantum interference.

\section{SAMPLE PREPARATION AND CHARACTERIZATION}

We attach fluorine adatoms to graphene covalently to produce stable fluorinated graphene. Graphene sheets are exfoliated from highly oriented pyrolytic graphite (HOPG) (Grade ZYA, GE) on $\mathrm{SiO}_{2}$ /doped $\mathrm{Si}$ substrates and fluorinated in a reactive ion etching chamber using a $\mathrm{CF}_{4}$ plasma at room temperature for 5-30 minutes, with a gas pressure of $100 \mathrm{mTorr}$ and a power setting of $5 \mathrm{~W} .{ }^{14}$ Here we work in the limit of very dilute fluorine coverage, where the low-energy electronic states are still dominated by the $\pi$ electrons of graphene. To remove fluorine, we anneal fluorinated graphene sheets in a tube furnace in the flow of forming gas $\left(90 \% \mathrm{Ar} / 10 \% \mathrm{H}_{2}\right)$ for $24 \mathrm{~h}$ at $365^{\circ} \mathrm{C}$. Figure 1 (a) shows the Raman spectra of DFG and defluorinated DFG samples. Fluorination produces a $D$ band. Its vanishing appearance after defluorination indicates a nearly full removal of fluorine adatoms with very few residue vacancies. In addition, defluorinated DFG has a mobility of $2000 \mathrm{~cm}^{2} / \mathrm{V} \mathrm{s}$, and shows Shubnikov-de Haas oscillations characteristic of high-quality single-layer graphene [Fig. 3(d)], thus confirming that (removable) fluorine adatoms are the dominant defect in our DFG samples. To determine the concentration and distribution of fluorine adatoms, scanning tunneling microscopy (STM) studies (Veeco Multimode) are carried out on DFG samples contacted by shadow mask-evaporated $\mathrm{Au}$ electrodes. The sample images, shown in Fig. 1(b), display the isolated, threefold symmetric, $(\sqrt{ } 3 \times \sqrt{ } 3) R 30^{\circ}$ superstructures characteristic of covalently bound fluorine. ${ }^{15}$ Images covering a total area of $0.04 \mu \mathrm{m}^{2}$ that hosts 900 defects imply an average F-F spacing of $7 \mathrm{~nm}$, which corresponds to a concentration $n_{\mathrm{F}}=2 \times 10^{12} / \mathrm{cm}^{2}$ and a highly dilute $\mathrm{F}: \mathrm{C}$ ratio of $1: 2000$.

\section{TRANSPORT MEASUREMENTS}

DFG samples are fabricated into field-effect transistors in Hall bar geometries using standard $e$-beam lithography [Fig. 2(a) inset]. ${ }^{16}$ Transport measurements are performed in a pumped ${ }^{4} \mathrm{He}$ cryostat equipped with a rotator and a 9 Tesla magnet, employing standard lock-in techniques and carefully limiting the excitation current to $0.5-50 \mathrm{nA}$ to avoid Joule heating. We present transport data on three fluorinated samples (called A, B, and C) and one defluorinated sample.

Figure 2(a) plots the sheet resistance vs. backgate voltage $R_{s}\left(V_{g}\right)$ on sample $\mathrm{A}$ at $B=0 \mathrm{~T}$ and selected temperatures. Electrons and holes exhibit approximately symmetric behavior, with a charge neutrality point of $+16 \mathrm{~V}$. Even with such a dilute F-adatom concentration, DFG is dramatically different from graphene. Pristine graphene is highly conductive with a weakly temperature-dependent resistance. In contrast, the resistance of DFG is much larger and displays an insulating temperature dependence. The resistance at the charge neutrality point increases by three orders of magnitude from $25 \mathrm{k} \Omega$ 

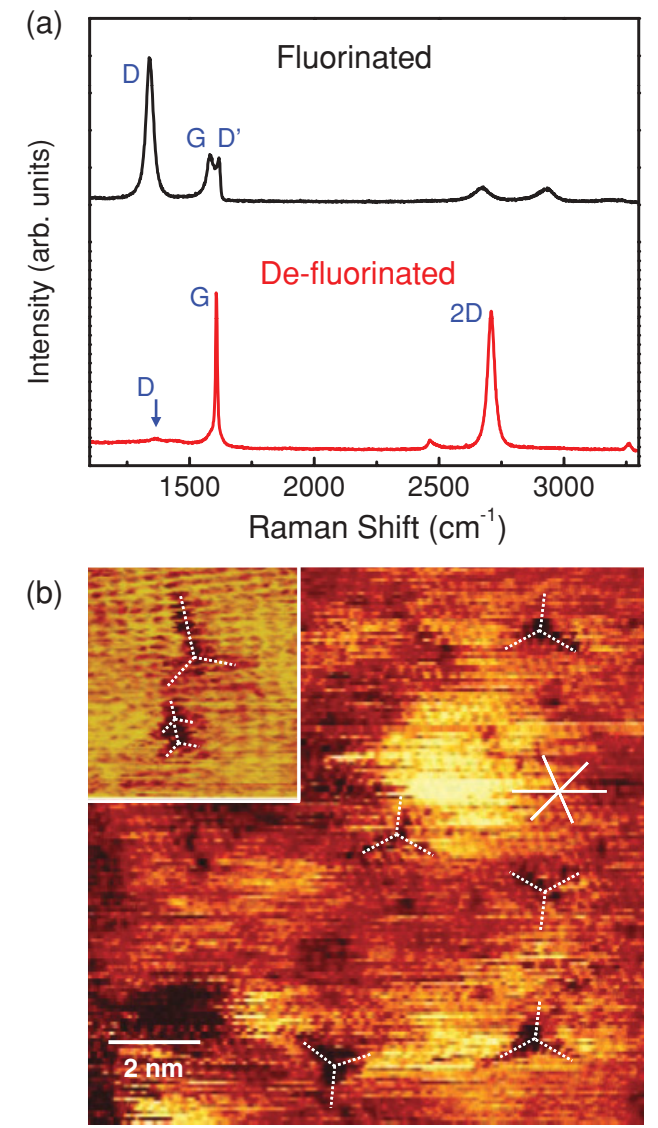

FIG. 1. (Color online) (a) Comparison of Raman spectra on fluorinated (upper trace) and defluorinated (lower trace) graphene using a 514-nm excitation wavelength. Fluorination conditions are identical for both samples. The Raman bands are marked in the figure. (b) A STM current image $(12 \mathrm{~nm} \times 12 \mathrm{~nm})$ of fluorinated graphene. $V_{\text {bias }}=20 \mathrm{mV}$. Color scale: $2 \mathrm{nA}$. The slowly varying background is owing to the height undulation of the $\mathrm{SiO}_{2}$ surface. The orientation of the unperturbed graphene lattice is marked by white solid lines. The white dotted lines mark the signature of isolated fluorine adatoms (Ref. 15). The fluorine distribution is inhomogeneous. F adatoms within $2 \mathrm{~nm}$ of each other are sometimes observed. Clusters are rare. Inset: three $\mathrm{F}$ adatoms close to each other with overlapping $\sqrt{3}$ superstructures. Color scale: $5 \mathrm{nA}$.

at $200 \mathrm{~K}$ to $2.5 \mathrm{M} \Omega$ at $5 \mathrm{~K}$. This insulating temperature dependence persists to higher carrier densities, but becomes weaker. At $n=3 \times 10^{12} / \mathrm{cm}^{2}$, the Hall mobility is merely $320 \mathrm{~cm}^{2} / \mathrm{V} \mathrm{s}$ at $200 \mathrm{~K}$. An analysis of $R_{S}(T)$ at different carrier densities reveals two distinct behaviors: strong localization (SL) and weak localization (WL), plus a transition between these two regimes. Examples are given in Fig. 2(b) for sample B. At low densities, $R_{S}(T)$ follows the temperature dependence of two-dimensional variable-range hopping (2D VRH): ${ }^{17}$

$$
\begin{gathered}
R \propto \exp \left(T_{0} / T\right)^{1 / 3} \text { and } \\
T_{0}=\frac{13.8}{k_{B} N\left(E_{\mathrm{F}}\right) \xi^{2}} \propto E_{b}^{1.5}=\left(E_{c}-E_{\mathrm{F}}\right)^{1.5} .
\end{gathered}
$$

Here $E_{c}, E_{\mathrm{F}}$, and $E_{b}$ are the mobility edge, Fermi level, and binding energy, respectively, while $N\left(E_{\mathrm{F}}\right), T_{0}$, and $\xi$ are the density of localized states at $E_{\mathrm{F}}$, the characteristic temperature,

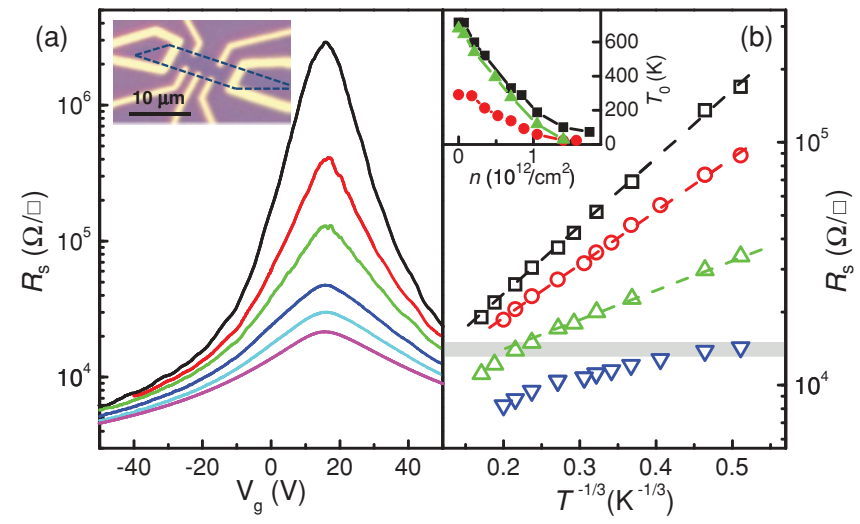

FIG. 2. (Color online) (a) Semi-log plot of $R_{s}$ vs backgate $V_{g}$ on sample A at $T=5,10,20,50,100$, and $200 \mathrm{~K}$ (top to bottom). The backgate dopes $7 \times 10^{10} / \mathrm{cm}^{2} V_{g}(\mathrm{~V})$ and the charge neutrality point occurs at $V_{g}=16 \mathrm{~V}$. Inset: Optical image of a DFG device with the graphene piece outlined. (b) Semilog plot of $R_{s}$ vs $T^{-1 / 3}$ on sample B at the charge neutrality point, $n=0.7,1.4$, and $2.5 \times 10^{12} / \mathrm{cm}^{2}$ (top to bottom). Dashed lines are fittings to the $2 \mathrm{D}$ VRH conduction, the slope of which yields $T_{0}$. The gray band corresponds to $\sim h / 2 e^{2}$. Inset: The $n$-dependent $T_{0}$ for samples A (black squares), B (red circles), and $\mathrm{C}$ (green triangles) in the strong localization regime.

and the localization length. $T_{0}$ is several hundred Kelvin at the charge neutrality point and decreases with increasing $n$, as shown in the inset of Fig. 2(b). In sample $\mathrm{B}, T_{0}$ reaches $24 \mathrm{~K}$ for $n \sim 1.4 \times 10^{12} / \mathrm{cm}^{2}$ and only data below $100 \mathrm{~K}$ are still described by the VRH model. As $n$ increases further, $R_{s}(T)$ deviates from the $\exp \left(T_{0} / T\right)^{1 / 3}$ expression at progressively lower temperature. For $n>2.5 \times 10^{12} / \mathrm{cm}^{2}$, $R_{S}(T)$ can no longer be described by Eq. (1), but instead exhibits a logarithmic temperature dependence consistent with weak localization. ${ }^{18}$ Similar density-driven strong localization to weak localization has also been observed in hydrogenated ${ }^{3}$ and ozone-damaged graphene., ${ }^{4,7}$

The crossover from $2 \mathrm{D} \mathrm{VRH}$ to $\ln T$ dependence is seen in all three DFG samples and indicates a transition in the vicinity of $R_{c} \sim 12 k \Omega / \square\left(\sim h / 2 e^{2}\right)$ [gray band in Fig. 2(b)] from a strongly localized insulator at low densities to a weakly localized metal at high densities. The density range where $R_{s}(T)$ crosses $R_{c}$ is roughly $n \sim 1.5-2.5 \times 10^{12} / \mathrm{cm}^{2}$ and is comparable to the average fluorine density $n_{\mathrm{F}}=2 \times$ $10^{12} / \mathrm{cm}^{2}$; this underlines the role of fluorine in this metalinsulator transition. Qualitatively similar transitions have been observed in two other defective graphene systems, although in neither case was the defect density known. ${ }^{3,4}$ Existing calculations show a negligible effect on conductivity for such a dilute adatom coverage. 6,7 Hence these fluorine adatoms cannot be viewed simply as random disorder.

The DFG samples also display a striking colossal negative magnetoresistance (MR) at the onset of strong localization. Figure 3(a) plots the normalized MR of sample A, $R_{S}(B) / R_{S}(0)$, in a perpendicular magnetic field for three densities in the weakly localized, transition, and strongly localized regimes. The negative MR in the weakly localized regime has a $B$ dependence that is well described by the theory of weak localization. ${ }^{18}$ The magnitude of the MR increases sharply as the density is lowered to reach the strongly 

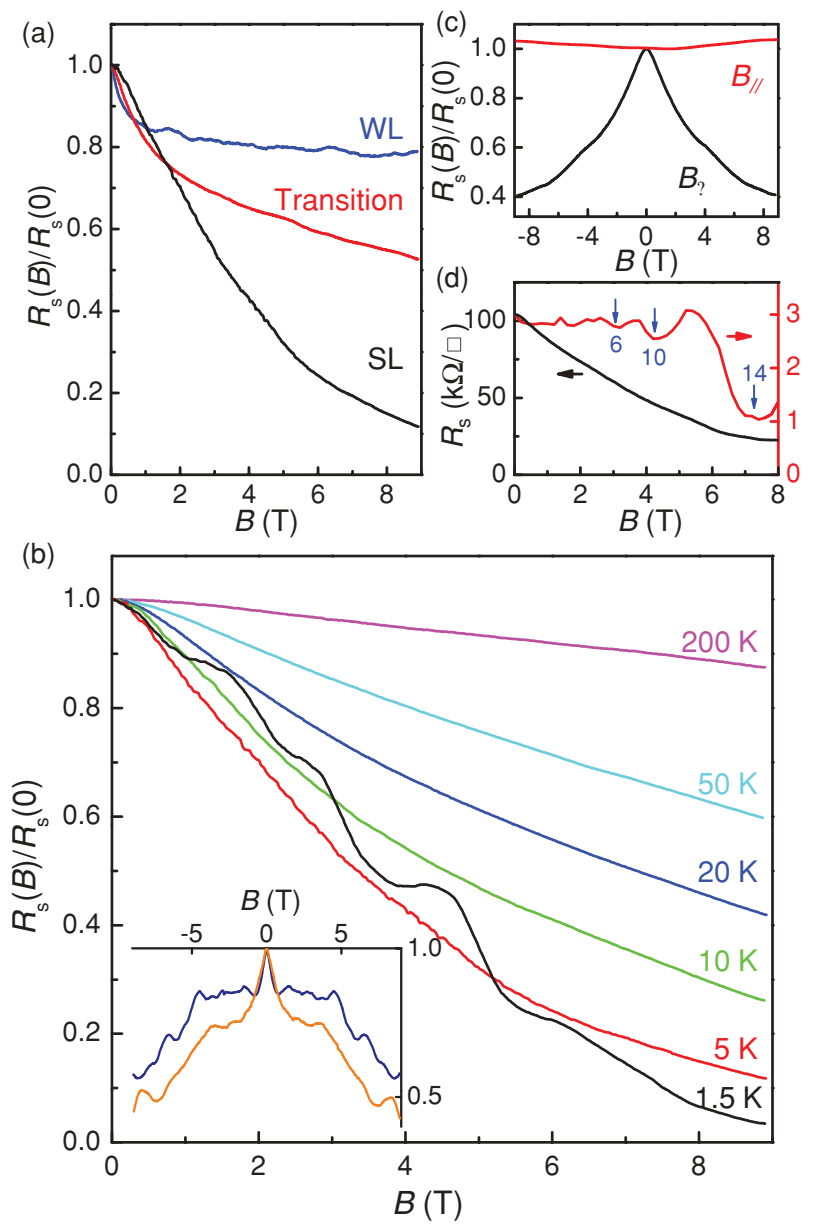

FIG. 3. (Color online) (a) Normalized MR in a perpendicular magnetic field, $R_{s}(B) / R_{s}(0)$, on sample A at $T=5 \mathrm{~K}$ and $n=0.8$ (black), 2.1 (red/gray), and $4.2 \times 10^{12} / \mathrm{cm}^{2}$ (blue/dark gray), labeled by their temperature dependence. (b) Normalized MR at $n=$ $0.8 \times 10^{12} / \mathrm{cm}^{2}$ and selected temperatures. Inset: Staircaselike MR observed on another sample (B) at $n=2.1$ (navy/dark gray, upper) and $1.4 \times 10^{12} / \mathrm{cm}^{2}$ (orange/gray, lower). $T=2 \mathrm{~K}$. (c) Normalized MR on sample $B$ at the charge neutrality point in perpendicular $\left(B_{\perp}\right)$ and in-plane $\left(B_{\|}\right)$magnetic fields showing a large anisotropy. (d) Comparison of magnetoresistance of DFG (black, scale on the left-hand side) and defluorinated DFG (red/gray, scale on the righthand side). For both samples, $n=1.05 \times 10^{12} / \mathrm{cm}^{2}$ and $T=5 \mathrm{~K}$. The defluorinated sample exhibits magnetoresistance oscillations of graphene with the filling factors marked in the figure.

localized regime. Figure 3(a) shows $R_{S}(B) / R_{S}(0)$ reaching 0.1 at $8.9 \mathrm{~T}$ with no sign of saturation. We observe up to a 40 -fold reduction in resistance in this regime and have verified that weak localization cannot describe the data. This strikingly large MR is the central result of this paper.

Figure 3(b) plots the temperature dependence of this MR for $n=8 \times 10^{11} / \mathrm{cm}^{2}$ in sample A. Above $5 \mathrm{~K}, R_{s}(B) / R_{s}(0)$ decreases smoothly with increasing $B$, quadratically at low $B$, and convex at higher $B$. The magnitude of the MR increases with decreasing temperature. Intriguingly, below $5 \mathrm{~K}$, a staircaselike dependence on magnetic field develops. The staircase becomes more pronounced at lower temperature and appears over a wide density range in the strongly localized and transition regimes for multiple samples [Fig. 3(b) inset]. To the
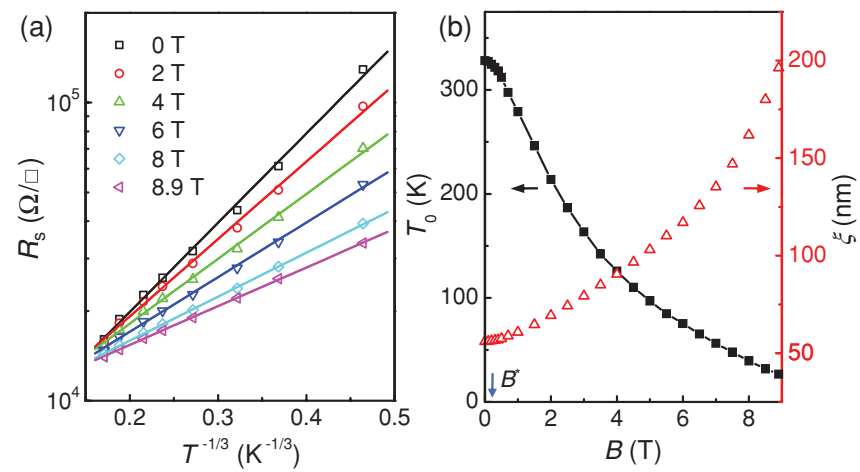

FIG. 4. (Color online) (a) $R_{s}$ vs $T^{-1 / 3}$ in a semilog plot for data shown in Fig. 3(b) at selected fields. Solid lines are fittings to the $2 \mathrm{D}$ VRH conduction, the slope of which yield $T_{0}$. (b) $T_{0}$ (scale on the left-hand side) and corresponding $\xi$ (scale on the right-hand side) vs $B$ from the data shown in (a). The blue arrow marks $B^{*}=0.21 \mathrm{~T}$.

best of our knowledge, such behavior has not been reported before in strongly localized systems.

To further illustrate the role of the magnetic field, we plot in Fig. 4(a) the sheet resistance $R_{S}(T)$ at fixed $B$ for the data shown in Fig. 3(b). $R_{s}(T)$ is well described by 2D VRH conduction at all $B$ fields, but the application of a magnetic field substantially reduces the degree of localization, with the extracted $T_{0}(B)$ decreasing from $330 \mathrm{~K}$ at $0 \mathrm{~T}$ to only $26 \mathrm{~K}$ at 8.9 T [Fig. 4(b)]. In Fig. 4(b), we also plot the $B$ dependence of the localization length $\xi(B)$ calculated using Eq. (1) and assuming a $B$-independent $N\left(E_{F}\right)$ approximated by the density of states of graphene at this density. The localization length increases from $56 \mathrm{~nm}$ at $0 \mathrm{~T}$ to $200 \mathrm{~nm}$ at $8.9 \mathrm{~T}$. Like the MR itself, it shows no signs of saturation.

\section{DISCUSSIONS}

We rule out magnetic contamination as the source of the observed MR because the same contaminants would have manifested in defluorinated DFG samples as well, in clear contradiction to data shown in Fig. 3(d). Strongly localized systems exhibit a rich variety of magnetoresistance, both positive and negative, but the majority of these effects are much smaller than what is observed here. ${ }^{19}$ Negative MR of this magnitude was seen in disordered $2 \mathrm{D}$ electron systems entering a reentrant quantum Hall state ${ }^{20}$ however, the $B$ dependence of that MR is very different from ours, and that phenomenon was attributed to a change of the density of states in the magnetic field. ${ }^{20,21}$ A Zeeman effect can also produce a large isotropic negative $\mathrm{MR}$ in systems near a metal-insulator transition. ${ }^{22}$ However, Fig. 3(c) shows that the magnetoresistance of sample $\mathrm{B}$ in an in-plane field is small $(<4 \%)$ and positive at $9 \mathrm{~T}$, in stark contrast to the large negative MR in the perpendicular field. This large anisotropy rules out the Zeeman effect and instead points to an orbital origin for the colossal negative MR. In strongly localized systems, two known quantum interference effects can produce a negative MR. The first considers the interference of forward hopping paths enclosing an area $A_{\mathrm{NSS}}=\left(r_{m}\right)^{3 / 2} \xi^{1 / 2}$, where $\xi$ is the localization length given in Eq. (1) and $r_{m}=\xi\left(T_{0} / T\right)^{1 / 3}$ is the hopping distance, with $r_{m} \geqslant \xi .^{23}$ This effect produces a negative MR of less than $10 \%$, which saturates near $B^{*}=\phi_{0} / A_{\mathrm{NSS}}$, where $\phi_{0}=h / e$. Using 
$r_{m}=\xi$ and the data of Fig. 4(b), we obtain an upper bound of $B^{*}<0.21 \mathrm{~T}$, which is clearly inconsistent with the magnitude of the MR and the observed lack of saturation up to $9 \mathrm{~T}$. In the second interference-based mechanism ${ }^{24}$ the magnetic field breaks time-reversal symmetry between forward and backward hopping paths (as for weak localization in metallic systems), thereby increasing $\xi$ and generating a negative MR. In quasi-1D systems, $\xi(B) / \xi(0)$ is predicted to saturate at a universal value of 2 for $B>B^{*}$. References 25 argue that $\xi(B) / \xi(0)$ need not be universal in higher dimensions and can be arbitrarily large; however, a quantitative theory in higher dimensions has yet to be developed. Experimental evidence of this effect is scant, ${ }^{24,26}$ with a doubling of $\xi$ shown in (3D) GaAs (Ref. 24) and a tenfold enhancement of conductance in $\mathrm{In}_{2} \mathrm{O}_{3-x}$ films. ${ }^{26}$ In both systems the MR saturates around the expected $B^{*}=\phi_{0} / \xi^{2}$. If the negative MR in our samples also results from time-reversal symmetry breaking, several notable discrepancies between our data and past experiments and theory must be reconciled. First, in our samples, $\left[\xi\left(B^{*}\right)-\xi(0)\right] / \xi(0)$ is only $\sim 0.5 \%$, compared to the order of unity predicted by theory ${ }^{24,25}$ and observed experimentally. ${ }^{24,26}$ Second, rather than saturating, $\xi(B)$ increases even more rapidly at $\sim 8.9 \mathrm{~T}$, where the magnetic length $l_{B}=(h / e B)^{1 / 2}=8.5 \mathrm{~nm}$ is much smaller than $\xi(0)$. Finally (and most importantly), the staircaselike MR at low temperatures suggests the existence of discrete energy levels, which couple to the magnetic field. This behavior has not been reported in any strongly localized systems before and is difficult to reconcile with existing theories. Interestingly, $l_{B}$ at $9 \mathrm{~T}$ is comparable to the mean free path $\left(l_{e}=5 \mathrm{~nm}\right.$ at $\left.200 \mathrm{~K}\right)$, which brings in the tantalizing possibility of the Hofstadter regime. ${ }^{24}$ If the large negative MR in our samples indeed originates from the suppression of quantum interference, it is quite extraordinary that nearly all of the resistance in our samples results from the interference of coherent scattering paths. This would be a remarkable realization of the Anderson localization of Dirac fermions, similar to the localization of light in disordered photonic crystals. ${ }^{27}$ The clean experimental geometry and the rich behavior exhibited by DFG present both challenges and opportunities to furthering the understanding of Anderson localization.

Motivated by the staircaselike MR and the strong resemblance of our data to those reported for colossal magnetoresistive manganites and dilute magnetic semiconductors on the insulating side of a ferromagnetic and metal-insulator transition, ${ }^{12,13}$ we consider a second scenario based on the formation of magnetic polarons that delocalize in a magnetic field. ${ }^{28}$ It has been predicted theoretically that magnetic moments of size $\sim \mu_{B}$ can be induced in graphene via adatoms, substitutes, or vacancies, ${ }^{8-10}$ although at present, there is no direct experimental evidence for magnetism in single-layer graphene (nor is direct magnetometric measurement possible owing to the small size of most graphene samples). In the magnetic polaron model, the exchange coupling $J \mathbf{S} \cdot \mathbf{S}$ between the localized electron spin and the surrounding local moments increases the bare binding energy of the localized electrons from $E_{b 0}$ to $E_{b}^{*}=E_{b 0}+E_{\mathrm{ex}} \cdot{ }^{28}$ The alignment of polarons in an external magnetic field increases their hopping probability, thereby reducing $E_{b}^{*}$ and unbinding the polarons. Although the dimensionality, mechanism of localization, and origin of the magnetic moments vary between magnetic semiconductors and graphene, the core concept of the magnetic polaron picture is appealing, given the concurrent onset of strong localization and colossal negative MR in our DFG samples. It should be emphasized also that this picture is different from the small polaron model, as applied to correlated oxides such as CMR manganites, ${ }^{13}$ since no local lattice distortion is expected in our system.

Applying the above concepts to our samples, we obtain a lower bound of $7 \mathrm{meV}$ for the enhanced binding energy $E_{b}^{*}$ at $B=0$, using $E_{b}^{*}>E_{a}(T)=d(\ln \rho) / d\left(k_{B} T\right)^{-1} \cdot{ }^{17}$ At $9 \mathrm{~T}, E_{b}^{*}$ is reduced by a factor of 5 , using the $T_{0}(B)$ data of Fig. 4(b) and Eq. (1). Hence the exchange enhancement dominates the polaron binding energy. In the polaron scenario, the absence of large MR in an in-plane magnetic field suggests a perpendicular orientation of the local moments. This observation is consistent with predictions of enhanced spin-orbit coupling of a few meV near adatoms as a result of $\sigma-\pi$ mixing. ${ }^{29}$ If true, the large magnetic anisotropy could be explored in spintronics applications to control the relaxation of spins injected in different orientations.

In fact, the two proposed scenarios do not exclude each other and may cooperate to give rise to the observed colossal negative MR. Independent of its origin, the magnitude of the phenomena provides the high sensitivity necessary for magnetic sensors. The staircaselike $B$ dependence seen in Fig. 3(b) at low temperatures is particularly intriguing. Interestingly, a staircaselike $B$ dependence is also seen in magnetization simulations of antiferromagnetically coupled local moments occupying opposite graphene sublattices. ${ }^{10}$ The nature and strength of the coupling between moments associated with $\mathrm{F}$ adatoms are currently unknown.

\section{CONCLUSION}

In conclusion, we demonstrate that fluorination provides a clean, reversible, and tunable approach to engineer defects and control the properties of graphene. Dilute fluorinated graphene exhibits a striking colossal anisotropic negative magnetoresistance and an unusual staircaselike magnetic-field dependence at low temperature. We propose two possible explanations in terms of quantum-interference-driven Anderson localization and/or adatom-induced magnetism. While the precise origin remains to be determined, this unique material system offers compelling opportunities to examine the role of strongly interacting defects in Dirac fermions.

\section{ACKNOWLEDGMENTS}

We are grateful for helpful discussions with B. Altshuler, A. Ayuela, A. H. Castro Neto, V. Crespi, M. Fuhrer, T. Rappoport, N. Samarth, P. Schiffer, J. Sofo, and B. Uchoa, and technical assistance from H. Gutierrez, J. Li, R. Misra, P. Joshi, M. Tian, and B. Wang. We thank N. Samarth and P. Schiffer for providing access to their Vecco multimode system. Work at Penn State is supported by NSF Grants CAREER No. DMR-0748604, NIRT No. ECS-0609243, and MRSEC No. DMR-0820404. The authors acknowledge use of facilities at the PSU site of NSF NNIN. 
${ }^{1}$ T. Stauber, N. M. R. Peres, and F. Guinea, Phys. Rev. B 76, 205423 (2007).

${ }^{2}$ T. O. Wehling, M. I. Katsnelson, and A. I. Lichtenstein, Chem. Phys. Lett. 476, 125 (2009).

${ }^{3}$ D. C. Elias, R. R. Nair, T. M. G. Mohiuddin et al., Science 323, 610 (2009).

${ }^{4}$ J. Moser, H. Tao, S. Roche, F. Alzina, C. M. Sotomayor Torres, and A. Bachtold, Phys. Rev. B 81, 205445 (2010).

${ }^{5}$ J.-H. Chen, W. G. Cullen, E. D. Williams et al., e-print arXiv:1004.3373 (2010).

${ }^{6}$ J. P. Robinson, H. Schomerus, L. Oroszlány, and V. I. Fal'ko, Phys. Rev. Lett. 101, 196803 (2008).

${ }^{7}$ N. Leconte, J. Moser, P. Ordejon et al., ACS Nano 4, 4033 (2010).

${ }^{8}$ M. A. H. Vozmediano, M. P. López-Sancho, T. Stauber, and F. Guinea, Phys. Rev. B 72, 155121 (2005).

${ }^{9}$ O. V. Yazyev and L. Helm, Phys. Rev. B 75, 125408 (2007); L. Brey, H. A. Fertig, and S. Das Sarma, Phys. Rev. Lett. 99, 116802 (2007); B. Uchoa, V. N. Kotov, N. M. R. Peres, and A. H. Castro Neto, ibid. 101, 026805 (2008); E. J. G. Santos, D. Sánchez-Portal, and A. Ayuela, Phys. Rev. B 81, 125433 (2010).

${ }^{10}$ T. G. Rappoport, B. Uchoa, and A. H. Castro Neto, Phys. Rev. B 80, 245408 (2009).

${ }^{11}$ O. V. Yazyev, Rep. Prog. Phys. 73, 056501 (2010).

${ }^{12}$ T. Dietl, Semicond. Sci. Technol. 17, 377 (2002).

${ }^{13}$ J. M. D. Coey, M. Viret, and S. von Molnar, Adv. Phys. 48, 167 (1999).

${ }^{14}$ K. Shoda, H. Kohno, Y. Kobayashi et al., J. Appl. Phys. 104, 113529 (2008).
${ }^{15}$ K. F. Kelly, E. T. Mickelson, R. H. Hauge et al., Proc. Natl. Acad. Sci. USA 97, 10318 (2000).

${ }^{16}$ X. Hong, K. Zou, and J. Zhu, Phys. Rev. B 80, 241415 (2009).

${ }^{17}$ B. I. Shklovskii and A. L. Efros, Electronic Properties of Doped Semiconductors (Springer, Berlin, 1984).

${ }^{18}$ E. McCann, K. Kechedzhi, V. I. Falko, H. Suzuura, T. Ando, and B. L. Altshuler, Phys. Rev. Lett. 97, 146805 (2006).

${ }^{19}$ S. L. di Vittorio, M. S. Dresselhaus, M. Endo, and T. Nakajima, Phys. Rev. B 43, 12304 (1991).

${ }^{20}$ H. W. Jiang, C. E. Johnson, and K. L. Wang, Phys. Rev. B 46, 12830 (1992).

${ }^{21}$ S. Kivelson, D.-H. Lee, and S.-C. Zhang, Phys. Rev. B 46, 2223 (1992).

${ }^{22}$ H. Fukuyama and K. Yoshida, J. Phys. Soc. Jpn. 46, 1522 (1979).

${ }^{23}$ O. Entin-Wohlman, Y. Imry, and U. Sivan, Phys. Rev. B 40, 8342 (1989).

${ }^{24}$ J.-L. Pichard, M. Sanquer, K. Slevin, and P. Debray, Phys. Rev. Lett. 65, 1812 (1990).

${ }^{25}$ J. P. Bouchaud, J. Phys. I (France) 1, 985 (1991); I. V. Lerner and Y. Imry, Europhys. Lett. 29, 49 (1995).

${ }^{26}$ A. Frydman and Z. Ovadyahu, Solid State Commun. 94, 745 (1995).

${ }^{27}$ T. Schwartz, G. Bartal, S. Fishman et al., Nature (London) 446, 52 (2007).

${ }^{28}$ A. Kaminski and S. Das Sarma, Phys. Rev. Lett. 88, 247202 (2002).

${ }^{29}$ A. H. Castro Neto and F. Guinea, Phys. Rev. Lett. 103, 026804 (2009). 